\title{
Lignicolous freshwater fungi from China and Thailand: Multi-gene phylogeny reveals new species and new records in Lophiostomataceae
}

\section{Bao DF ${ }^{1,2,3}$, Su HY ${ }^{1}$, Maharachchikumbura $\mathrm{SSN}^{4}$, Liu JK${ }^{4}$, Nalumpang $\mathrm{S}^{3}$, Luo $\mathbf{Z L}^{1 *}$ and Hyde $\mathrm{KD}^{2}$}

${ }^{1}$ College of Agriculture and Biological Sciences, Dali University, Dali 671003, Yunnan, P.R. China

${ }^{2}$ Center of Excellence in Fungal Research, Mae Fah Luang University, Chiang Rai 57100, Thailand

${ }^{3}$ Department of Entomology and Plant Pathology, Faculty of Agriculture, Chiang Mai University, Chiang Mai 50200, Thailand

${ }^{4}$ School of Life Science and Technology, University of Electronic Science and Technology of China, Chengdu 611731, P.R. China

Bao DF, Su HY, Maharachchikumbura SSN, Liu JK, Nalumpang S, Luo ZL, Hyde KD 2019 Lignicolous freshwater fungi from China and Thailand: Multi-gene phylogeny reveals new species and new records in Lophiostomataceae. Mycosphere 10(1), 1080-1099, Doi $10.5943 /$ mycosphere/10/1/20

\begin{abstract}
We are investigating the diversity of lignicolous freshwater fungi from China and Thailand. In this study, six collections of Lophiostomataceae-like taxa were made from freshwater habitats in China and Thailand, of which three are identified as existing species Biappendiculispora japonica, Neovaginatispora fuckelii and Vaginatispora armatispora. While, the other collections are recognized as new species, Flabellascoma aquaticum sp. nov., F. fusiforme sp. nov. and Sigarispora clavata sp. nov. and these are introduced herein based on the morphological characters and multigene phylogenetic analyses of combined LSU, SSU, ITS, TEF1 $-\alpha$ and RPB2 sequence data. Detailed descriptions and illustrations of these six species are provided.
\end{abstract}

Key words - 3 new species - Dothideomycetes - Pleosporales - sexual morphs - phylogeny taxonomy

\section{Introduction}

Lignicolous freshwater fungi grow on submerged woody debris in freshwater streams, ponds, lakes and tree hollows (Hyde 1995, Wong et al. 1998, Ho et al. 2002). They play an important role in ecosystem functioning and nutrient recycling of woody material (Palmer et al. 1997, Wong et al. 1998, Hyde and Goh 1998, Bucher et al. 2004). Lignicolous freshwater fungi are highly diverse and probably sensitive to environmental change and global warming (Hyde et al. 2016). We are studying the diversity of lignicolous freshwater fungi along the north-south gradient in the Asian/Australasian region (Hyde et al. 2016). By collecting these data, we are contributing to the knowledge of biogeographical diversity of fungi in freshwater habitats.

The family Lophiostomataceae was previously reported as "Lophiostomeae" by Nitschke (1869). Subsequently, Saccardo (1883) formally established Lophiostomataceae and placed it in 
Pleosporales. Species mostly occur as saprobes in terrestrial, freshwater and marine environments (Tibpromma et al. 2017, Hashimoto et al. 2018, Hyde et al. 2019). Their intergeneric relationships have been discussed by Kodsueb et al (2006) and Wang et al. (2007) and recently by Hashimoto et al. (2018). Members of this family are characterized by immersed to erumpent, carbonaceous to coriaceous ascomata with rounded or slit-like ostioles, mostly clavate asci and 1 to multi-septate, hyaline to dark brown ascospores with terminal appendages or mucilaginous sheaths (Hyde et al. 2013, 2017, Ariyawansa et al. 2015, Liu et al. 2015, Thambugala et al. 2015). Thambugala et al. (2015) revised the classification of this family, provided a backbone tree and accepted 16 genera. Wanasinghe et al. (2018) introduced a new genus, Muritestudina and Hashimoto et al. (2018) introduced seven genera in Lophiostomataceae. Presently, 24 genera are accepted in Lophiostomataceae (Wijayawardene et al. 2017, Hashimoto et al. 2018, Wanasinghe et al. 2018, Hyde et al. 2019).

During a survey of lignicolous freshwater fungi along the north-south gradient in the Asian/Australian region (Hyde et al. 2016), six lophiostomataceous lignicolous freshwater taxa were collected from Tibet and Yunnan provinces, China and southern Thailand. Three new species, viz. Flabellascoma aquaticum, F. fusiforme and Sigarispora clavata are introduced based on morphological characters and multi-gene phylogenetic analysis. Detailed descriptions and illustrations of the new species and records are also provided.

\section{Materials \& Methods}

\section{Isolation and morphological examination}

Submerged decaying wood samples were collected from Tibet and Yunnan provinces, China and Sai khu waterfall, Thailand, and brought to the laboratory in plastic bags. The samples were incubated in plastic boxes lined with moistened tissue paper at room temperature for 1 week. Sample examination and morphological studies followed protocols outlined previously (Luo et al. 2018).

The fungal species present on the substrates were isolated using a single spore culture technique following the method described in Chomnunti et al. (2014). Germinating ascospores were transferred aseptically to fresh potato dextrose agar (PDA) media with antibiotics and incubated at room temperature for 2-4 weeks. Cultures were grown for 1-2 months and morphological characters such as colour, colony shape, texture and asexual morphs were recorded and checked after 30-60 days. The cultures were deposited in Mae Fah Luang University Culture Collection (MFLUCC) and Kunming Institute of Botany culture collection (KUMCC), herbarium specimens (dry wood with the fungi and slides) were deposited in Mae Fah Luang University (MFLU). Faces of fungi and Index Fungorum numbers were registered as detailed in Jayasiri et al. (2015) and in Index Fungorum (http://www.indexfungorum.org/names/nam-es.asp) respectively.

\section{DNA extraction, PCR amplification and sequencing}

Genomic DNA was extracted from fungal mycelium grown on PDA or MEA at $25{ }^{\circ} \mathrm{C}$ using EZ gene ${ }^{\mathrm{TM}}$ Fungal gDNA Kits (GD2416) according to the manufacturer's instructions. The regions of large subunit rRNA (LSU), internal transcribed spacers (ITS), small subunit rRNA (SSU), translation elongation factor (TEF1- $\alpha$ ) and RNA polymerase II subunit 2 (RPB2) were amplified using the primer pairs LR0R/LR7 (Vilgalys \& Hester 1990), ITS5/ITS4, NS1/ NS4 (White et al. 1990), 983F/2218R and fRPB2-5F/fRPB2-7cR (Liu et al. 1999) respectively. The amplification reactions were performed in $25 \mu \mathrm{L}$ of PCR mixtures containing $12.5 \mu \mathrm{l}$ of $2 \times$ Power Taq PCR Master Mix (a premix and ready to use solution, including 0.1 Units/ $\mu 1$ Taq DNA Polymerase, $500 \mu \mathrm{m}$ dNTP 
Mixture each (dATP, dCTP, dGTP, dTTP), $20 \mathrm{mM}$ Tris- $\mathrm{HCl} \mathrm{pH} 8.3,100 \mathrm{Mm} \mathrm{KCl}, 3 \mathrm{mM} \mathrm{MgCl} 2$, stabilizer and enhancer), $1 \mu \mathrm{l}$ of each primer, $1 \mu \mathrm{l}$ DNA template and $9.5 \mu 1$ deionized water. The PCR profile for the ITS, LSU, SSU and TEF1- $\alpha$ gene regions as follows: initial denaturation for $94{ }^{\circ} \mathrm{C}$ for 3 minutes, followed by 35 cycles of denaturation at $94{ }^{\circ} \mathrm{C}$ for 30 seconds, annealing at $56{ }^{\circ} \mathrm{C}$ for 50 seconds, elongation at $72{ }^{\circ} \mathrm{C}$ for 1 minute and a final extension at $72{ }^{\circ} \mathrm{C}$ for 10 minutes. The RPB2 gene region was amplified with an initial denaturation of $95^{\circ} \mathrm{C}$ for $5 \mathrm{~min}$, followed by 40 cycles of denaturation at $95{ }^{\circ} \mathrm{C}$ for $1 \mathrm{~min}$, annealing at $54{ }^{\circ} \mathrm{C}$ for 40 seconds, elongation at $72{ }^{\circ} \mathrm{C}$ for 90 seconds, and the final extension at $72{ }^{\circ} \mathrm{C}$ for $10 \mathrm{~min}$. Purification and sequencing of PCR products were carried out using the above-mentioned PCR primers at Beijing Tsingke Biological Engineering Technology and Services Co., Ltd. (Beijing, P.R. China)

\section{Sequence alignment and phylogenetic analyses}

Sequence data for relevant strains were downloaded from GenBank following recent publications (Table 1) (Thambugala et al. 2015, Hashimoto et al. 2018, Wanasinghe et al. 2018, Hyde et al. 2019). Consensus sequences were assembled using BioEdit and aligned using MAFFT v.7.110 online program (http://mafft.cbrc.jp/alignment/server/) (Katoh \& Standley 2013) and manually edited using BioEdit v7.2.3 (Hall 1999). The phylogenetic analyses were performed using Randomized Accelerated Maximum Likelihood (RAxML) and Bayesian analyses. Phylogeny website tool "ALTER" (Glez-Peña et al. 2010) was used to convert the alignment fasta file to Phylip format and Maximum likelihood (ML) analysis was performed using the CIPRES Science Gateway v.3.3 (http:// www.phylo.org/portal2/; Miller et al. 2010) using RAxML v.8.2.8 as part of the "RAxML-HPC2 on XSEDE" tool (Stamatakis 2006, Stamatakis et al. 2008). All free model parameters were estimated by RAxML with ML estimates of 25 per site rate categories. The final ML search was conducted using the GTRGAMMA + I substitution model.

Bayesian analysis was performed by MrBayes v3.1.2 (Ronquist et al. 2012). The model of evolution was estimated by using MrModeltest 2.2 (Nylander 2004). Posterior probabilities (Rannala \& Yang 1996) were performed by Markov Chain Monte Carlo Sampling (BMCMC) in MrBayes v. 3.1.2. Six simultaneous Markov Chains were run for 1 million generations and trees were sampled every 100th generation (resulting in 10,000 trees). The first 2,000 trees representing the burn-in phase of the analyses were discarded and the remaining 8,000 (post burning) trees used for calculating posterior probabilities $(\mathrm{PP})$ in the majority rule consensus tree.

Table 1 Isolates and sequences used in this study. The newly generated sequences are indicated in red and ex-type strains are indicated in bold.

\begin{tabular}{llccccc}
\hline \multirow{2}{*}{ Taxa } & \multirow{2}{*}{ Strain number } & \multicolumn{5}{c}{ GenBank accession numbers } \\
\cline { 3 - 7 } & & SSU & ITS & LSU & TEF1-a & RPB2 \\
\hline Alpestrisphaeria terricola & SC-12 & JX985749 & JN662930 & JX985750 & - & - \\
Alpestrisphaeria jonesii & GAAZ 54-1 & KX687755 & KX687757 & KX687753 & KX687759 & - \\
Alpestrisphaeria jonesii & GAAZ 54-2 & KX687756 & KX687758 & KX687754 & KX687760 & - \\
Biappendiculispora japonica & KT 573 & AB618686 & LC001728 & AB619005 & LC001744 & - \\
Biappendiculispora japonica & KT 686-1P & AB618687 & LC001729 & AB619006 & LC001745 & - \\
Biappendiculispora japonica & MFLUCC 17-2450 & MN304834 & MN304829 & MN328900 & - & - \\
Capulatispora sagittiformis & KT 1934 & AB618693 & AB369268 & AB369267 & LC001756 & - \\
Coelodictyosporium muriforme & MFLUCC 13-0351 & KP899127 & KP899136 & KP888641 & KR075163 & - \\
\hline
\end{tabular}


Table 1 Continued.

\begin{tabular}{|c|c|c|c|c|c|c|}
\hline \multirow{2}{*}{ Taxa } & \multirow{2}{*}{ Strain number } & \multicolumn{5}{|c|}{ GenBank accession numbers } \\
\hline & & SSU & ITS & LSU & TEF1- $\alpha$ & RPB2 \\
\hline $\begin{array}{l}\text { Coelodictyosporium } \\
\text { pseudodictyosporium }\end{array}$ & MFLUCC 13-0451 & - & KR025858 & KR025862 & - & - \\
\hline Crassiclypeus aquaticus & CBS 143641 & LC312470 & LC312499 & LC312528 & LC312557 & LC312586 \\
\hline Crassiclypeus aquaticus & CBS 143642 & LC312471 & LC312500 & LC312529 & LC312558 & LC312587 \\
\hline Dimorphiopsis brachystegiae & СРC 22679 & - & KF777160 & KF777213 & - & - \\
\hline Flabellascoma minimum & CBS 143645 & LC312474 & LC312503 & LC312532 & LC312561 & LC312590 \\
\hline Flabellascoma minimum & CBS 143646 & LC312475 & LC312504 & LC312533 & LC312562 & LC312591 \\
\hline Flabellascoma aquaticum & KUMCC15-0258 & MN304832 & MN304827 & MN274564 & MN328898 & MN328895 \\
\hline Flabellascoma cycadicola & CBS 143644 & LC312473 & LC312502 & LC312531 & LC312560 & LC312589 \\
\hline Flabellascoma fusiforme & MFLUCC 18-1584 & - & MN304830 & MN274567 & MN328902 & - \\
\hline Guttulispora crataegi & MFLUCC 13-0442 & KP899125 & KP899134 & KP888639 & KR075161 & - \\
\hline Guttulispora crataegi & MFLUCC 14-0993 & KP899126 & KP899135 & KP888640 & KR075162 & - \\
\hline Lentistoma bipolare & CBS 143651 & LC312483 & LC312512 & LC312541 & LC312570 & LC312599 \\
\hline Lentistoma bipolare & CBS 143652 & LC312484 & LC312513 & LC312542 & LC312571 & LC312600 \\
\hline Leptoparies palmarum & CBS 143653 & LC312485 & LC312514 & LC312543 & LC312572 & LC312601 \\
\hline Lophiohelichrysum helichrysi & MFLUCC 15-0701 & KT333437 & KT333435 & KT333436 & KT427535 & - \\
\hline Lophiopoacea paramacrostoma & MFLUCC 11-0463 & KP899122 & - & KP888636 & - & - \\
\hline Lophiopoacea winteri & KT 740 & AB618699 & JN942969 & AB619017 & LC001763 & JN993487 \\
\hline Lophiopoacea winteri & KT 764 & AB618700 & JN942968 & AB619018 & LC001764 & JN993488 \\
\hline Lophiostoma caulium & CBS 623.86 & GU296163 & - & GU301833 & - & GU371791 \\
\hline Lophiostoma crenatum & CBS 629.86 & DQ678017 & - & DQ678069 & DQ677912 & DQ677965 \\
\hline Lophiostoma heterosporum & CBS 644.86 & AY016354 & GQ203795 & AY016369 & DQ497609 & DQ497615 \\
\hline Lophiostoma macrostomoides & CBS 123097 & FJ795482 & - & FJ795439 & GU456277 & FJ795458 \\
\hline Lophiostoma macrostomum & KT 635 & AB521731 & AB433275 & AB433273 & LC001752 & JN993484 \\
\hline Lophiostoma quadrinucleatum & GKM 1233 & - & - & GU385184 & GU327760 & - \\
\hline Lophiostoma semiliberum & KT 828 & AB618696 & JN942970 & AB619014 & LC001759 & JN993489 \\
\hline Lophiostoma alpigenum & GKM 1091b & - & - & GU385193 & GU327758 & - \\
\hline Lophiostoma multiseptatum & JCM17668 & AB618684 & LC001726 & AB619003 & LC001742 & \\
\hline $\begin{array}{l}\text { Neotrematosphaeria } \\
\text { biappendiculata }\end{array}$ & KT 1124 & GU205256 & - & GU205227 & - & - \\
\hline $\begin{array}{l}\text { Neotrematosphaeria } \\
\text { biappendiculata }\end{array}$ & KT 975P & GU205254 & - & GU205228 & - & - \\
\hline Neovaginatispora fuckelii & MFLUCC 17-1334 & MN304833 & MN304828 & MN274565 & MN328899 & MN328896 \\
\hline Neovaginatispora fuckelii & CBS 101952 & FJ795496 & - & DQ399531 & - & FJ795472 \\
\hline Neovaginatispora fuckelii & KH 161 & AB618689 & LC001731 & AB619008 & LC001749 & - \\
\hline Neovaginatispora fuckelii & KT 634 & AB618690 & LC001732 & AB619009 & LC001750 & - \\
\hline $\begin{array}{l}\text { Parapaucispora } \\
\text { pseudoarmatispora }\end{array}$ & KT 2237 & LC100018 & LC100021 & LC100026 & LC100030 & - \\
\hline Paucispora quadrispora & KT 843 & AB618692 & LC001734 & AB619011 & LC001755 & - \\
\hline Paucispora versicolor & KH 110 & LC001721 & AB918731 & AB918732 & $\mathrm{LC001760}$ & - \\
\hline
\end{tabular}


Table 1 Continued.

\begin{tabular}{|c|c|c|c|c|c|c|}
\hline \multirow{2}{*}{ Taxa } & \multirow{2}{*}{ Strain number } & \multicolumn{5}{|c|}{ GenBank accession numbers } \\
\hline & & SSU & ITS & LSU & TEF1- $\alpha$ & RPB2 \\
\hline Paucispora quadrispora & KH 448P & LC001720 & LC001733 & LC001722 & LC001754 & - \\
\hline Platystomum actinidiae & KT 521 & JN941375 & JN942963 & JN941380 & LC001747 & JN993490 \\
\hline Platystomum compressum & MFLUCC 13-0343 & KP899129 & - & KP888643 & KR075165 & - \\
\hline Platystomum crataegi & MFLUCC 14-0925 & KT026113 & KT026117 & KT026109 & KT026121 & - \\
\hline Platystomum rosae & MFLUCC 15-0633 & KT026115 & - & KT026111 & KT026119 & - \\
\hline Platystomum salicicola & MFLUCC 15-0632 & KT026114 & KT026118 & KT026110 & - & - \\
\hline $\begin{array}{l}\text { Pseudolophiostoma } \\
\text { obtusisporum }\end{array}$ & CBS 143941 & LC312490 & LC312519 & LC312548 & LC312577 & LC312606 \\
\hline $\begin{array}{l}\text { Pseudolophiostoma } \\
\text { obtusisporum }\end{array}$ & CBS 143658 & LC312491 & LC312520 & LC312549 & LC312578 & LC312607 \\
\hline Pseudolophiostoma tropicum & CBS 143659 & LC312492 & LC312521 & LC312550 & LC312579 & LC312608 \\
\hline Pseudolophiostoma tropicum & CBS 143660 & LC312493 & LC312522 & LC312551 & LC312580 & LC312609 \\
\hline Pseudolophiostoma vitigenum & HH 26930 & AB618697 & LC001735 & AB619015 & LC001761 & - \\
\hline Pseudolophiostoma vitigenum & HH 26931 & AB618698 & LC001736 & AB619016 & LC001762 & - \\
\hline $\begin{array}{l}\text { Pseudopaucispora } \\
\text { brunneospora }\end{array}$ & CBS 143661 & LC312494 & LC312523 & LC312552 & LC312581 & 312610 \\
\hline $\begin{array}{l}\text { Pseudoplatystomum } \\
\text { scabridisporum }\end{array}$ & BCC 22835 & 831 & - & 5844 & 857 & GU4798 \\
\hline $\begin{array}{l}\text { Pseudoplatystomum } \\
\text { scabridisporum }\end{array}$ & $\mathrm{BCC}$ & GQ925832 & - & GQ925845 & GU4 & GU479829 \\
\hline Sigarispora clavata & MFLUCC 18-1316 & MN304835 & - & MN274566 & MN328901 & - \\
\hline Sigarispora arundinis & KT 651 & AB618680 & JN942965 & AB618999 & LC001738 & JN993486 \\
\hline Sigarispora caryophyllacearum & MFLUCC 17-0749 & MG829176 & MG828964 & MG829076 & MG829238. & - \\
\hline Sigarispora caudata & KT 530 & AB618681 & LC001723 & AB619000 & LC001739 & - \\
\hline Sigarispora caulium & MFLUCC 15-0036 & MG829177 & MG828965 & MG829077 & MG829239 & - \\
\hline Sigarispora coronillae & MFLUCC 14-0941 & KT026116 & KT026120 & KT026112 & - & - \\
\hline Sigarispora junci & MFLUCC 14-0938 & MG829178 & MG828966 & MG829078 & - & - \\
\hline Sigarispora medicaginicola & MFLUCC 17-0681 & MG829179 & MG828967 & MG829079 & - & - \\
\hline Sigarispora muriformis & MFLUCC 13-0744 & KY501 & KY496740 & KY496719 & - & - \\
\hline Sigarispora ononidis & MFLUCC 14-0613 & KU243126 & KU243128 & KU243125 & KU243127 & - \\
\hline Sigarispora ravennica & MFLUCC 14-0005 & KP698415 & KP698413 & KP698414 & - & - \\
\hline Sigarispora rosicola & MFLU 15-1888 & MG829180 & MG828968 & MG829080 & MG829240 & - \\
\hline Sigarispora scrophulariae & MFLUCC 17-0689 & MG829181 & MG828969 & MG829081 & - & - \\
\hline Sigarispora thymi & MFLU 15-2131 & MG829182 & MG828970 & MG829082 & MG829241 & - \\
\hline Teichospora rubriostiolata & TR 7H & - & KU601590 & KU601590 & KU601609 & KU601599 \\
\hline Teichospora trabicola & C $134 \mathrm{E}$ & - & KU601591 & KU601591 & KU601601 & KU601600 \\
\hline Vaginatispora amygdali & KT2248 & LC312495 & LC312524 & LC312553 & LC312582 & LC312640 \\
\hline Vaginatispora amygdali & MFLUCC 18-1586 & MK085057 & MK085055 & MK085059 & MK087657 & - \\
\hline Vaginatispora appendiculata & MFLUCC 16-0314 & KU743219 & KU743217 & KU743218 & KU743220 & - \\
\hline Vaginatispora aquatica & MFLUCC 11-0083 & KJ591575 & KJ591577 & KJ591576 & - & - \\
\hline
\end{tabular}


Table 1 Continued.

\begin{tabular}{lllllll}
\hline \multirow{2}{*}{ Taxa } & \multirow{2}{*}{ Strain number } & \multicolumn{4}{c}{ GenBank accession numbers } \\
\cline { 3 - 7 } & & SSU & ITS & LSU & TEF1-a & RPB2 \\
\hline Vaginatispora armatispora & MFLUCC 18-0247 & MK085058 & MK085056 & MK085060 & MK087658 & MK087669 \\
\hline Vaginatispora armatispora & HKTLCC1562 & - & AF383955 & - & - & - \\
\hline Vaginatispora armatispora & MFLUCC 18-0213 & MN304831 & MN304826 & MN274563 & MN328897 & MN328894 \\
Vaginatispora microarmatispora & MTCC 12733 & MF142594 & MF142592 & MF142593 & MF142595 & MF142596 \\
Vaginatispora scabrispora & KT2443 & LC312496 & LC312525 & LC312554 & LC312583 & LC312612 \\
\hline
\end{tabular}

\section{Results}

\section{Phylogenetic analyses}

The combined LSU, SSU, ITS, TEF1- $\alpha$ and RPB2 dataset comprised 82 taxa of Lophiostomataceae, with Teichospora rubriostiolata (TR7) and Teichospora trabicola (C134) as the outgroup taxa. The dataset comprised 4,188 characters after alignment including gaps (LSU: 1-826; SSU: 827-1767; ITS: 1768-2283; TEF1- $\alpha$ : 2284-3177; RPB2: 3178-4188). The RAxML analysis of the combined dataset yielded a best scoring tree (Fig. 1) with a final ML optimization likelihood value of -28080.438440 . The matrix had 1551 distinct alignment patterns, with $26.56 \%$ undetermined characters or gaps. Estimated base frequencies were as follows: $\mathrm{A}=0.250308, \mathrm{C}=$ $0.245041, \mathrm{G}=0.267629, \mathrm{~T}=0.237021$; substitution rates $\mathrm{AC}=1.632712, \mathrm{AG}=4.315873, \mathrm{AT}=$ 1.313155, $\mathrm{CG}=1.501540, \mathrm{CT}=9.264809, \mathrm{GT}=1.000000$; gamma distribution shape parameter $\alpha$ $=0.180969$. Support values for maximum likelihood (ML) above than $75 \%$ and Bayesian posterior probabilities (PP) greater than 0.95 are given at the nodes.

The phylogenetic analyses showed that newly isolates of Vaginatispora armatispora, Biappendiculispora japonica and Neovaginatispora fuckelii clustered with V. armatispora (MFLUCC 18-0247, HKTLCC 1562), B. japonica (KT 573, KT 686) and N. fuckelii (KT 634, KH 161, CBS 101952), respectively, with strong statistical support (100 ML/1.00 PP, 98 ML/1.00 PP and $100 \mathrm{ML} / 1.00 \mathrm{PP}$, respectively).

The novel species Sigarispora clavata grouped with members of the genus Sigarispora as a sister taxon to $S$. caudata with strong statistical support (76 ML/1.00 PP). The other new species, Flabellascoma aquaticum and $F$. fusiforme clustered together within the genus Flabellascoma, but they formed distinct lineages with significant statistical support (96 ML/1.00 PP and 100 ML/1.00 $\mathrm{PP}$, respectively).

Flabellascoma aquaticum D.F. Bao, Z.L. Luo, K.D. Hyde \& H.Y. Su, sp. nov.

Fig. 2

Index Fungorum number: IF 556720; Facesoffungi number: FoF 06212

Etymology - Referring to the aquatic habitat

Holotype - MFLU 19-0992

Saprobic on submerged decaying wood. Sexual morph: Ascomata 280-440 $\mu \mathrm{m}$ high, 260-390 $\mu \mathrm{m}$ diam $(\bar{x}=327 \times 360 \mu \mathrm{m}, \mathrm{n}=8)$, immersed, scattered, subglobose, dark brown to black, with a long, black neck. Ostiole crest-like, dark brown to black, elongated, composed of brown to black cells. Peridium 25-52 $\mu \mathrm{m}$ wide, uniform, comprising 2 zones, outer layers composed several dark brown cells, inner zone composed of 5-7 layers of rectangular, hyaline to pale brown cells of textura 


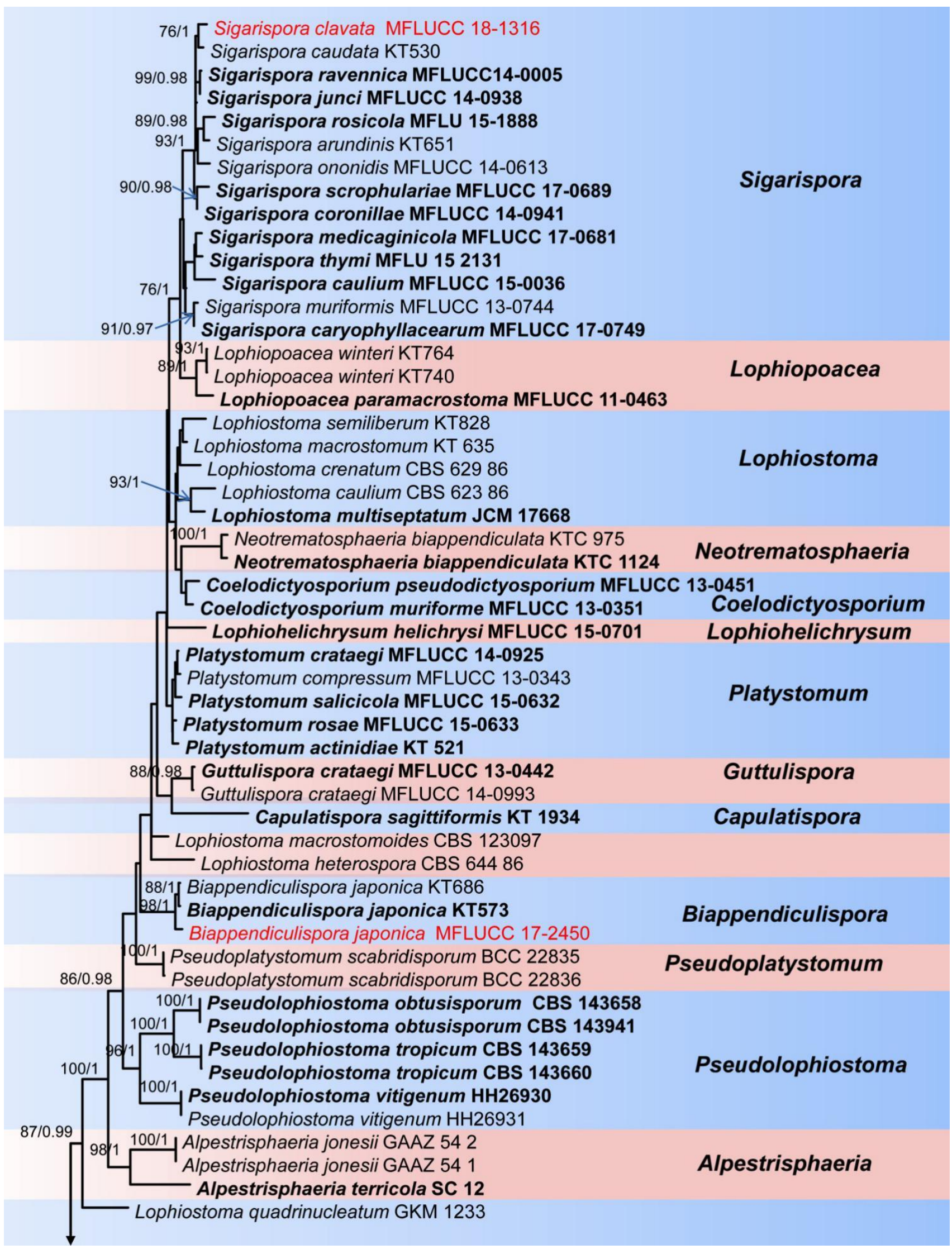

Figure 1 - Phylogenetic tree based on RAxML analyses of combined LSU, SSU, ITS, TEF1- $\alpha$ and RPB2 sequence data. Maximum likelihood bootstrap $>75 \%$ and Bayesian posterior probabilities > 0.95 (BYPP) are indicated at the nodes. The ex-type strains are shown in bold and the newly obtained 
isolates are shown in red. The tree is rooted with Teichospora rubriostiolata (TR7) and Teichospora trabicola (C134).

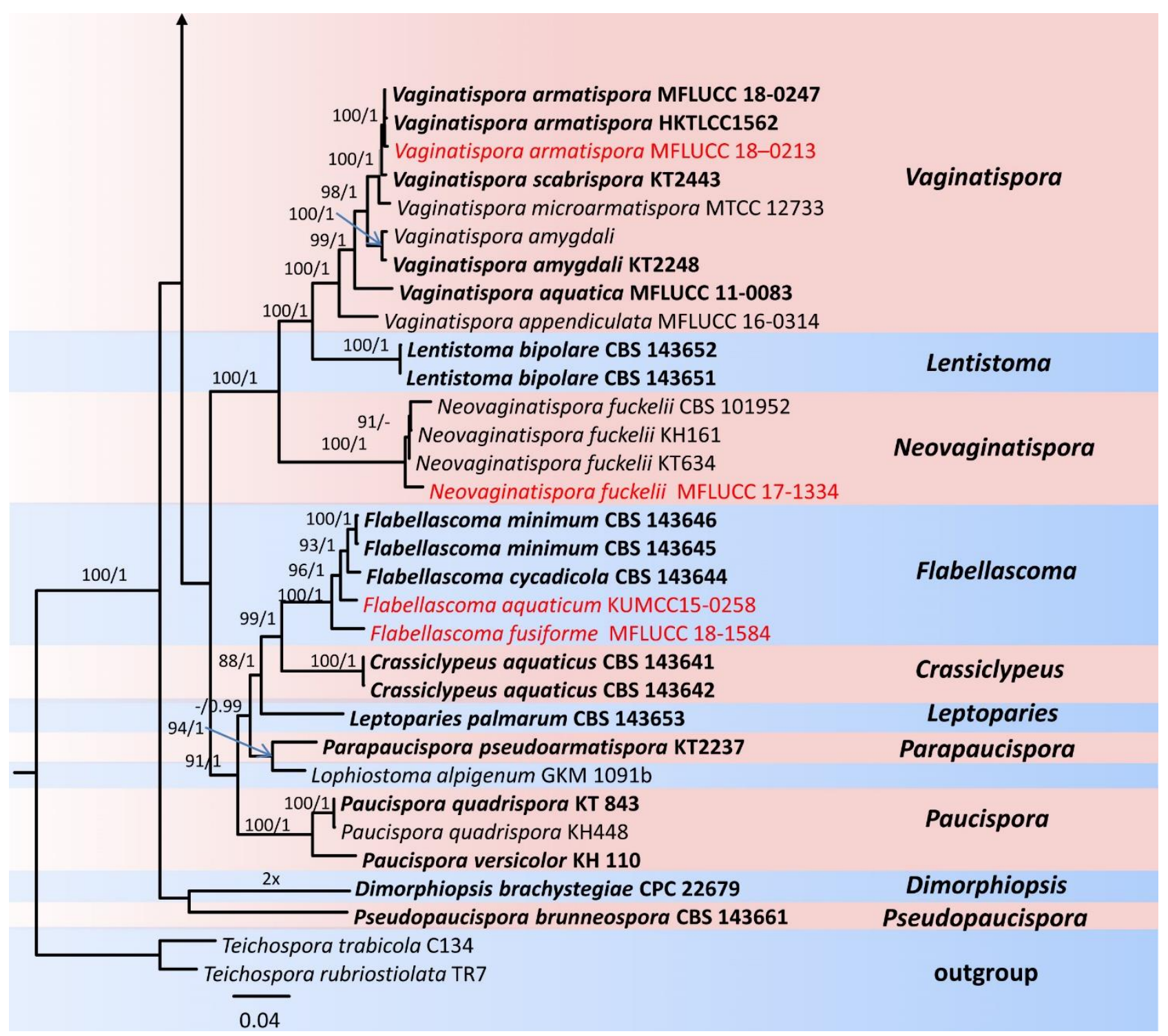

Figure 1 - Continued.

angularis and globulosa. Hamathecium comprising numerous, 1.2-2 $\mu \mathrm{m}$ wide, cellular pseudoparaphyses, septate, branched and anastomosed. Asci 48-72 $\times 8-9 \mu \mathrm{m}(\bar{x}=60 \times 8.6 \mu \mathrm{m}, \mathrm{n}$ $=20$ ), 8 -spored, bitunicate, fissitunicate, cylindrical-clavate, short pedicellate, apically rounded with an ocular chamber. Ascospores 16-18 $\times 4.3-5.3 \mu \mathrm{m}(\bar{x}=17 \times 5 \mu \mathrm{m}, \mathrm{n}=30)$, fusiform with narrow and obtuse ends, hyaline, uniseptate, slightly constricted at the septum, 4-guttules, 2 middle ones larger than end ones, with a narrow sheath. Appendages drawn out from sheath at both of ends (4.7$7 \mu \mathrm{m}$ wide, $\mathrm{n}=30$ ), hyaline, unbranched. Asexual morph: Undetermined.

Material examined - CHINA, Yunnan Province, saprobic on decaying wood submerged in Dulong River (2753'51.50" N, 98²0'13.10" E), May 2015, Z.L. Luo, S-390 (MFLU 19-0992, holotype), ex-type living culture, KUMCC 15-0258. 

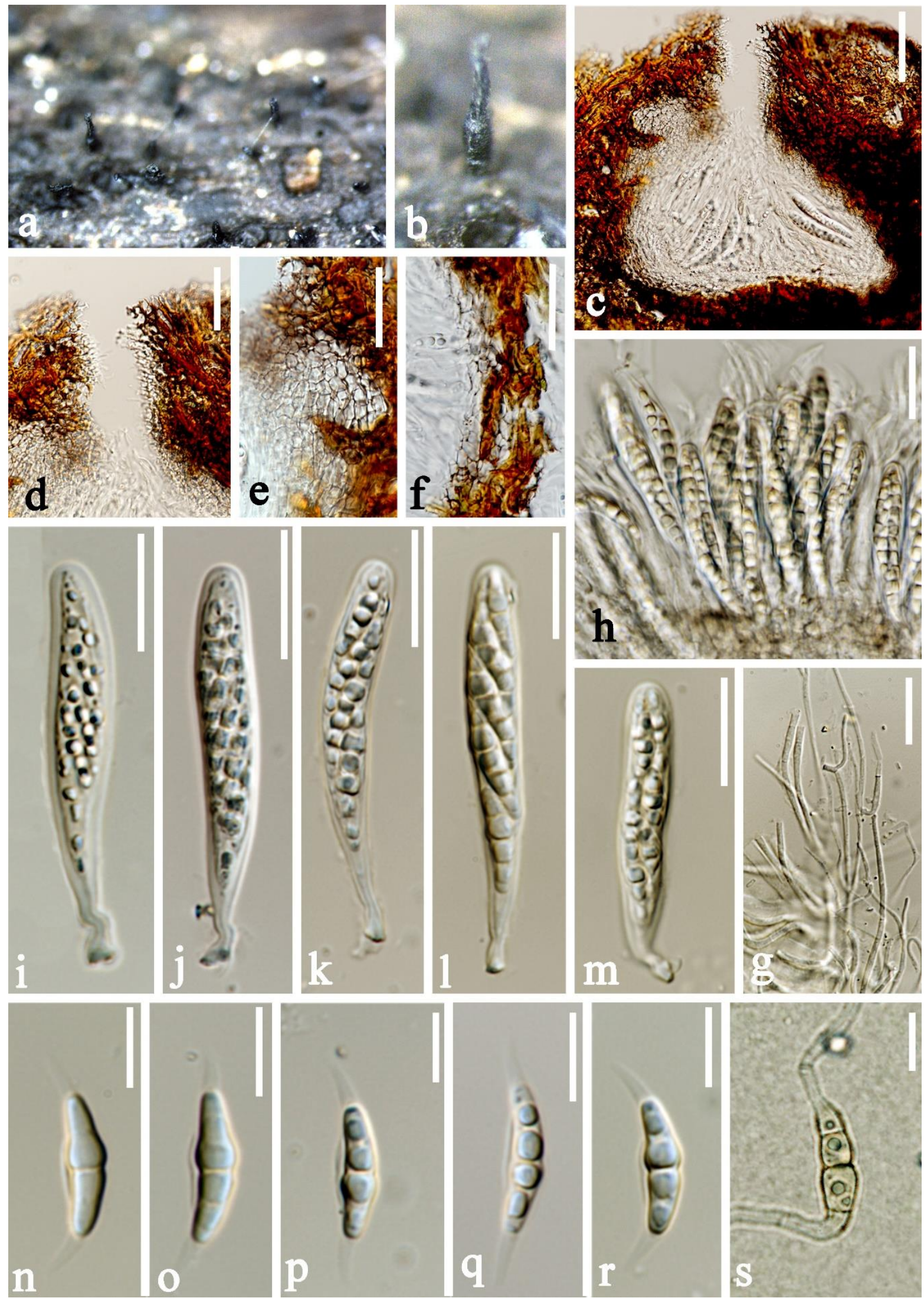

Figure 2 - Flabellascoma aquaticum (MFLU 19-0992, holotype). a Ascomata on submerged wood. b Neck of ascoma. c Section of ascoma. d Ostiolar neck of ascoma. e, f Peridium of ascomata. $g$ Pseudoparaphyses. $\mathrm{h}-\mathrm{m}$ Asci. $\mathrm{n}-\mathrm{r}$ Ascospore. $\mathrm{s}$ Germinating ascospore. Scale bars: $\mathrm{c}=50 \mu \mathrm{m}, \mathrm{d}-\mathrm{f}=$ $30 \mu \mathrm{m}, \mathrm{g}-\mathrm{m}=20 \mu \mathrm{m}, \mathrm{n}-\mathrm{s}=10 \mu \mathrm{m}$. 
Notes - Flabellascoma species have similar shaped asci and ascospores. $F$. cycadicola and $F$. minimum are distinguishable from each other based on ascospore size. F. aquaticum differs from $F$. cycadicola in ascus and ascospore size (48-72 $\times 8-9$ vs. $67.5-88 \times 9-12 \mu \mathrm{m}$ and $16-18 \times 4.3-5.3$ vs. 17-23 $\times$ 4.5-7 $\mu \mathrm{m})$. Moreover, $F$. aquaticum can be morphologically distinguished from $F$. cycadicola and $F$. minimum based on ascomata characters. $F$. aquaticum has ascomata with a cylindrical, long, black neck (Fig. 2b). Whereas, ascomata of $F$. cycadicola and $F$. minimum have short, elongated, crest-like, ostiolar neck. In the phylogenetic analysis, F. aquaticum and F. fusiforme grouped with other existing Flabellascoma species, but formed distinct lineages, and thus are distinct species (Fig. 1).

Flabellascoma fusiforme D.F. Bao, Z.L. Luo, K.D. Hyde \& H.Y. Su, sp. nov.

Fig. 3

Index Fungorum number: IF 556721; Facesoffungi number: FoF 06213

Etymology - referring to the fusiform ascospores.

Holotype - MFLU 19-0993

Saprobic on submerged decaying wood. Sexual morph: Ascomata 310-420 $\mu \mathrm{m}$ high, 320-380 $\mu \mathrm{m}$ diam $(\bar{x}=358 \times 345 \mu \mathrm{m}, \mathrm{n}=5)$, scattered, immersed, subglobose, dark brown to black. Ostiole crest-like, central, with a reduced crest and a pore-like opening, composed dark brown to black cells. Peridium 25-50 $\mu \mathrm{m}$ wide, composed two strata, outer stratum comprising brown to dark brown, thick-walled cells, inner stratum composed of several layers with lightly pigmented to hyaline cells. Hamathecium comprising 1.5-3 $\mu \mathrm{m}$ wide, septate, branched, cellular pseudoparaphyses. Asci 66-80 $\times 10-12 \mu \mathrm{m}(\bar{x}=72.8 \times 10.8 \mu \mathrm{m}, \mathrm{n}=20), 8$-spored, bitunicate, fissitunicate, cylindrical-clavate with a furcate pedicel, apically rounded with a broad ocular chamber. Ascospores $15-18 \times 4-5 \mu \mathrm{m}(\bar{x}=$ $16.6 \times 4.7 \mu \mathrm{m}, \mathrm{n}=30$ ), fusiform, with narrow and obtuse to acute ends, hyaline, uniseptate, 4-guttules, 2 middle ones larger than end ones, constricted at septum, smooth, with a thin sheath, appendages drawn out from sheath at both of ends $(5.4-8 \mu \mathrm{m}$ wide, $\mathrm{n}=30)$, hyaline, unbranched. Asexual morph: Undetermined.

Material examined - CHINA, Yunnan Province, saprobic on decaying wood submerged in Nujiang River (27³7'29.92" N, 9845'38.10" E), October 2016, Z.L. Luo, S-1583 (MFLU 19-0993, holotype), ex-type culture, MFLUCC 18-1584

Notes - Currently, two species are accepted in Flabellascoma. In this study, we introduce two additional species in this genus. The new species Flabellascoma fusiforme fits well with the morphological characters of Flabellascoma, such as immersed ascomata, bitunicate, fissitunicate, cylindrical-clavate asci and fusiform, hyaline, 1-septate ascospores with a narrow bipolar sheath. We herein compare the morphological differences between new species Flabellascoma fusiforme with other Flabellascoma species.

Flabellascoma fusiforme is phylogenetically close to $F$. aquaticum (Fig. 1). However, Flabellascoma fusiforme can be easily distinguished from $F$. aquaticum by its ascomatal shape and ascus size. Flabellascoma fusiforme has ascomata with a short, elongated, crest-like ostiolar neck (Fig. 3a, b, c), while the ascomata in F. aquaticum has a cylindrical, long, black neck (Fig. 2b) and the asci of $F$. fusiforme are larger than $F$. aquaticum $(66-80 \times 10-12$ vs. $48-72 \times 8-9 \mu \mathrm{m})$.

Flabellascoma fusiforme can be distinguished from $F$. cycadicola in having smaller ascospores (15-18 $\times 4-5$ vs. $17-23 \times 4.5-7 \mu \mathrm{m})$ and longer ascus pedicel. In addition, both the ascospores ends of $F$. cycadicola are narrower than $F$. fusiforme. Furthermore, $F$. fusiforme differs from $F$. minimum in its shape of ascomata and ascospores; $F$. fusiforme has subglobose ascomata and straight ascospores. However, F. minimum ascomata are ellipsoidal to lageniform and ascospores are slightly curved. 

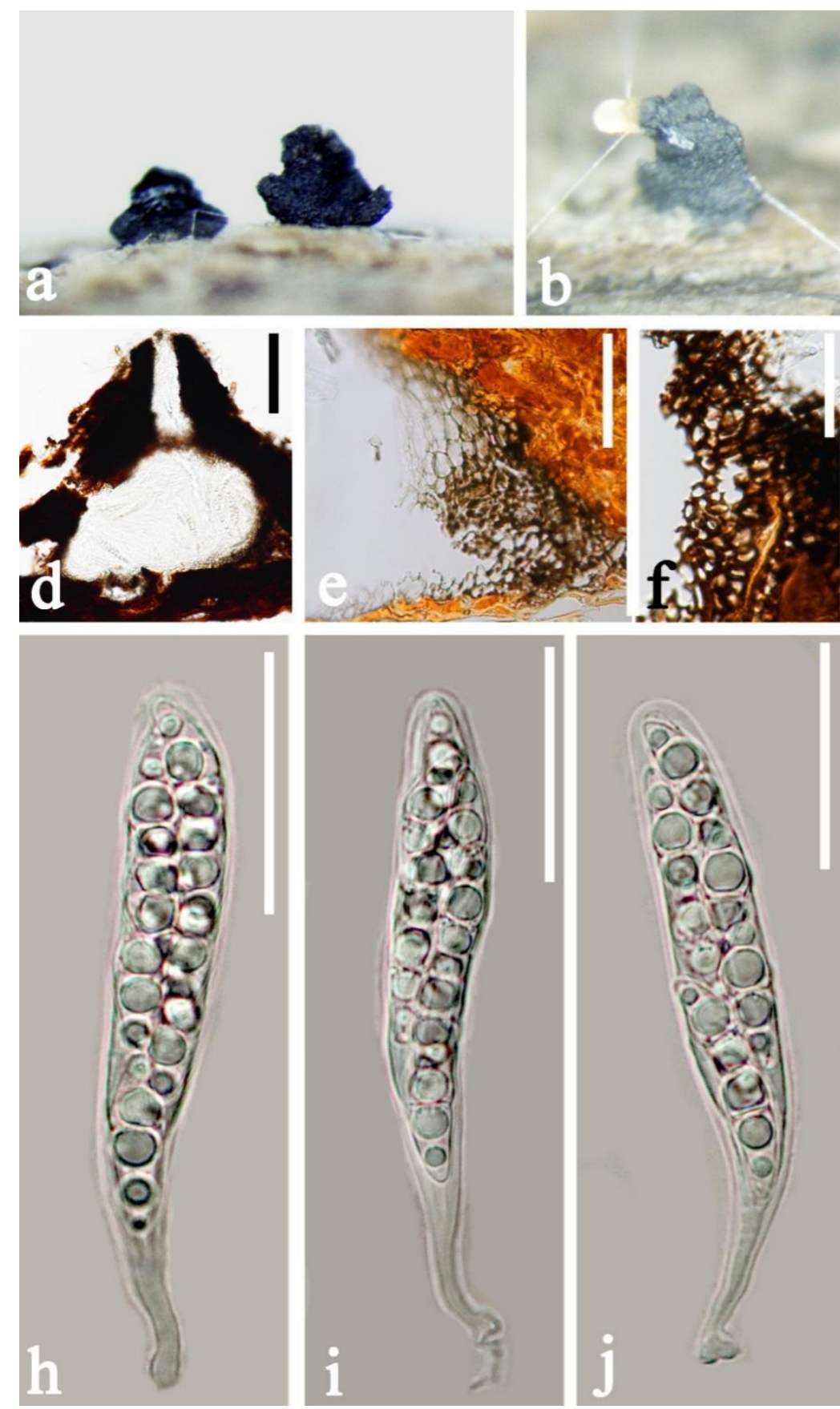
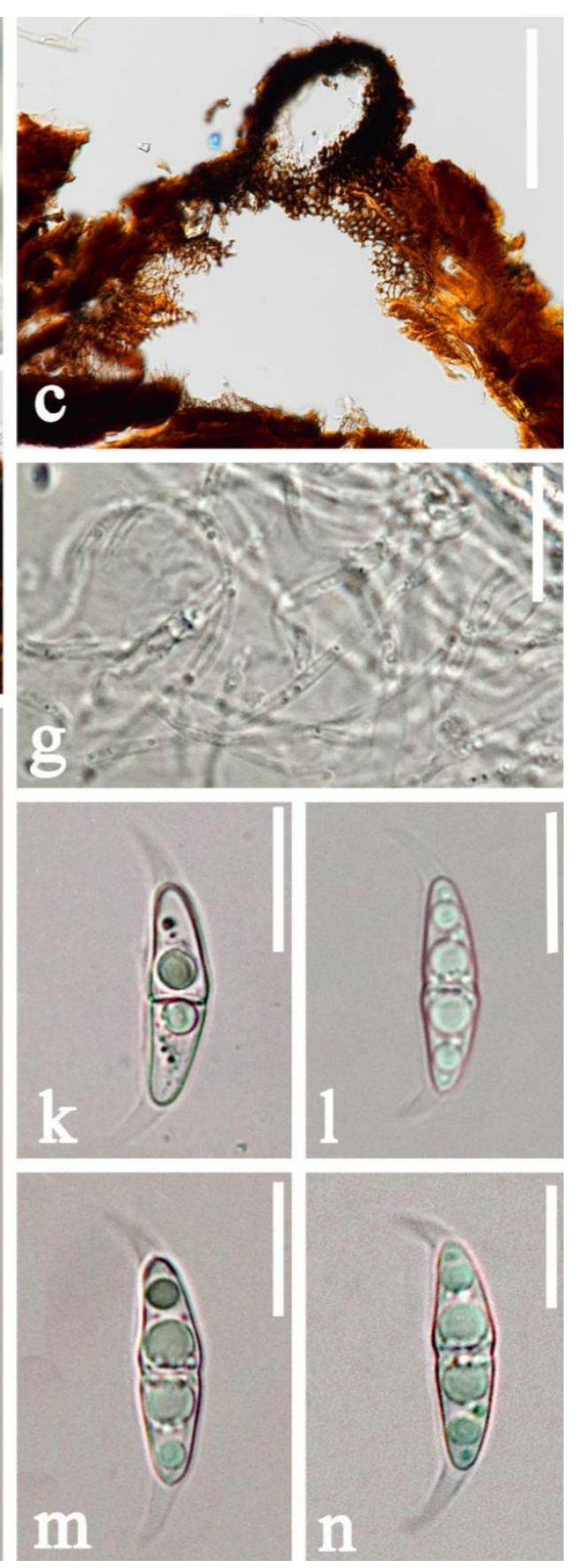

Figure 3 - Flabellascoma fusiforme (MFLU 19-0993, holotype). a-c Ascomata on submerged wood. c, d Section of ascoma. e, f Peridium of ascomata. g Pseudoparaphyses. $\mathrm{h}-\mathrm{j}$ Asci. $\mathrm{k}-\mathrm{n}$ Ascospore. Scale bars: $\mathrm{c}, \mathrm{d}=100 \mu \mathrm{m}, \mathrm{e}=50 \mu \mathrm{m}, \mathrm{f}, \mathrm{h}-\mathrm{j}=30 \mu \mathrm{m}, \mathrm{g}=20 \mu \mathrm{m}, \mathrm{k}-\mathrm{n}=10 \mu \mathrm{m}$.

Sigarispora clavata D.F. Bao, Z.L. Luo, K.D. Hyde \& H.Y. Su, sp. nov.

Fig. 4

Index Fungorum number: IF 556722; Facesoffungi number: FoF 06214

Etymology - Referring to the clavate ascospores.

Holotype - MFLU 19-0994

Saprobic on submerged decaying wood in freshwater habitats. Sexual morph: Ascomata 327 $470 \mu \mathrm{m}$ high, $280-460 \mu \mathrm{m}$ diam $(\bar{x}=380 \times 400 \mu \mathrm{m}, \mathrm{n}=5)$, semi-immersed to immersed, subglobose, coriaceous, black, ostiolate. Ostiole slit-like, variable in shape, with a crest-like apex and a pore-like opening, plugged by gelatinous tissue, made up of lightly pigmented, pseudoparenchymatous cells. 

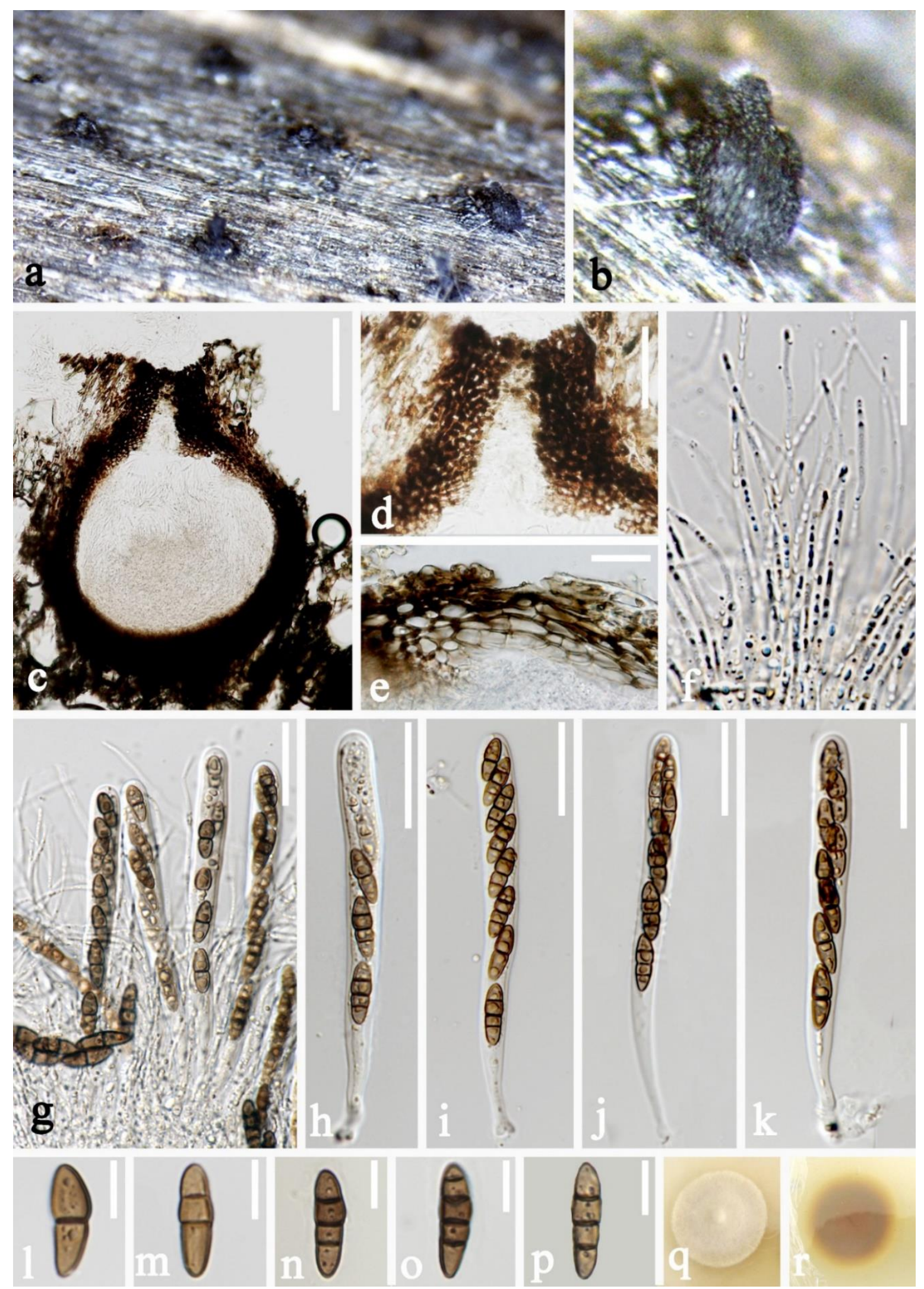

Figure 4 - Sigarispora clavata (MFLU 19-0994, holotype). a, b Ascomata on submerged wood. c Section of ascoma. d Ostiolar neck of ascoma. e Peridium. f Pseudoparaphyses. g-k Asci. 1-p Ascospore. $q$, $r$ culture on PDA. Scale bars: $\mathrm{c}=100 \mu \mathrm{m}, \mathrm{d}=50 \mu \mathrm{m}, \mathrm{e}, \mathrm{g}-\mathrm{k}=30 \mu \mathrm{m}, \mathrm{f}=20 \mu \mathrm{m}, \mathrm{l}-\mathrm{p}$ $=10 \mu \mathrm{m}$. 
Peridium 32-55 $\mu \mathrm{m}$ wide, comprises a single stratum, with brown to dark brown cells of textura globulosa and textura angularis. Hamathecium comprising 1.5-2 $\mu \mathrm{m}$ wide, filiform, hyaline, septate, guttulate, smooth cellular pseudoparaphyses. Asci 91-117 ×9-12 $\mu \mathrm{m}(\bar{x}=104 \times 11 \mu \mathrm{m}, \mathrm{n}=20)$, cylindrical, round at the apex, bitunicate, long pedicellate, with a small ocular chamber at the apex. Ascospores $13-17 \times 4-6 \mu \mathrm{m}(\bar{x}=15.4 \times 5.3 \mu \mathrm{m}, \mathrm{n}=30)$, uni to bi-seriate, overlapping, ellipsoidal to clavate, with obtuse ends, straight or slightly curved, hyaline when young, dark brown to yellowish brown at maturity, 1-4-septate, slightly constricted at the septum, guttules, smooth walled. Asexual morph: Undetermined.

Material examined - CHINA, Tibet Autonomous Region ( $\left.30^{\circ} 15^{\prime} 08.02^{\prime \prime} \mathrm{N}, 82^{\circ} 58^{\prime} 27.77^{\prime \prime} \mathrm{E}\right)$, on submerged decaying wood, May 2017, Z.L. Luo, 2XZ A 1-2-1, S-1483 (MFLU 19-0994, holotype), ex-type culture, MFLUCC 18-1316.

Notes - Species of Sigarispora are characterized by immersed, papillate, ostiolate ascomata, a peridium of pseudoparenchymatous cells, bitunicate, fissitunicate asci and ellipsoidal-fusiform or muriform ascospores, with or without sheaths, and appendages. Our species fits well within the species concept of genus Sigarispora. However, our species can be distinguished from other species of Sigarispora by its 1-4-septate, ellipsoidal to clavate ascospores with obtuse ends.

In our study, Sigarispora clavata is sister to $S$. caudata with good support (76 ML/1.00 PP). Sigarispora clavata shares similar morphological characteristics with $S$. caudate in having immersed to semi-immersed, coriaceous ascomata, bitunicate, cylindrical asci with ocular chamber and brown to dark brown ascospores. However, S. clavata differs from $S$. caudate in ascomatal size (380-400 $\times$ 280-460 vs. $145-210 \times 210-305 \mu \mathrm{m})$ and ascospores shape, septations and size (1-4 vs. 4-6 septate, 13-17 × 4-6 vs. $23.5-34.5 \times 5.5-7 \mu \mathrm{m}$, respectively), $S$. clavata has ellipsoidal to clavate ascospores with obtuse ends, whereas ascospores of $S$. caudata are fusiform with narrow and acute ends.

Biappendiculispora japonica Thambug., Wanas., Kaz. Tanaka \& K.D. Hyde, in Thambugala et al., Fungal Diversity, 74: 214 (2015)

Fig. 5

Index Fungorum Number: IF551529; Facesoffungi number: FoF01097

Saprobic on submerged decaying wood. Sexual morph: Ascomata 330-420 $\mu \mathrm{m}$ high, 240-300 $\mu \mathrm{m}$ diam $(\bar{x}=383 \times 276 \mu \mathrm{m}, \mathrm{n}=5)$, semi-immersed to immersed, solitary to gregarious, subglobose, papillate, coriaceous, black. Ostiole slit-like, central, brown to dark brown. Peridium 15-25 $\mu \mathrm{m}$ wide composed one stratum, with several layers of brown to dark brown, cells of textura angularis, cells towards the inside lighter, outside is darker. Hamathecium comprising numerous, branched, septate, guttulate, cellular pseudoparaphyses, embedded in a gelatinous matrix. Asci 108-130 × 15-18 $\mu \mathrm{m}$ $(\bar{x}=119 \times 16.4 \mu \mathrm{m}, \mathrm{n}=20), 8$-spored, bitunicate, fissitunicate, cylindric-clavate, with short pedicel, rounded at the apex, with an ocular chamber. Ascospores $29.8-39 \times 6-8 \mu \mathrm{m}(\bar{x}=34.2 \times 7 \mu \mathrm{m}, \mathrm{n}=$ 30 ), overlapping uni to bi-seriate, fusiform with acute ends, mostly curved, 7-8-septate, constricted at the septa, guttulate, hyaline when young, becoming yellowish to brown at maturity, smooth, with appendages at both ends. Asexual morph: Undetermined.

Material examined - CHINA, Yunnan Province, Dali city, Midu County, saprobic on decaying wood submerged in a stream, May 2015, H.W. Shen S-787 (DLU 787), living culture, MFLUCC $17-2450$.

Notes - Biappendiculispora japonica was introduced by Tanaka \& Harada (2003) and it was previously placed in the genus Lophiostoma. Subsequently, Thambugala et al. (2015) showed that $L$. japonica grouped as a sister clade to Pseudolophiostoma. Therefore, Thambugala et al. (2015) introduced a new genus Biappendiculispora to accommodate Lophiostoma japonica. Morphologically, our fresh collection fits well with B. japonica, such as immersed, coriaceous 
ascomata, bitunicate, cylindric-clavate asci with an ocular chamber and hyaline to brown, fusiform ascospores with acute ends. Phylogenetic analysis showed that our isolate clustered together with $B$. japonica with strong bootstrap support (98 ML/1.00 PP) (Fig. 1). Therefore, we identify our collection as Biappendiculispora japonica and it is a new record from China which was previously reported in Japan from terrestrial habitats.
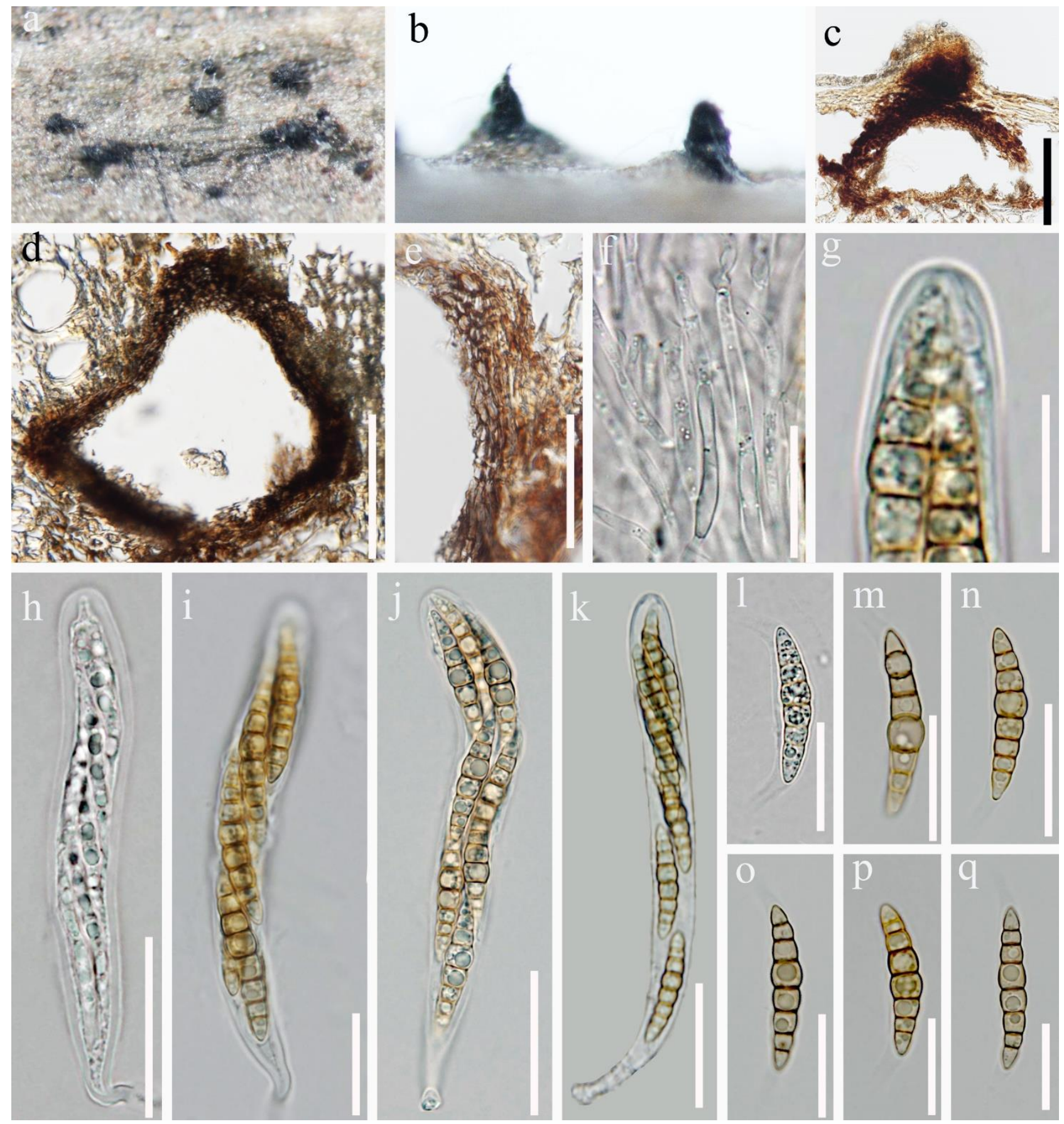

Figure 5 - Biappendiculispora japonica (DLU 787) a, b Ascomata on submerged wood. c, d Section of ascoma. e Peridium. f Pseudoparaphyses. g Ascus apex. h-k Asci. l-q Ascospore. Scale bars: c, d $=100 \mu \mathrm{m}, \mathrm{e}=50 \mu \mathrm{m}, \mathrm{f}-\mathrm{g}, \mathrm{l}-\mathrm{q}=20 \mu \mathrm{m}, \mathrm{f}=20 \mu \mathrm{m}, \mathrm{h}-\mathrm{k}=30 \mu \mathrm{m}$.

Neovaginatispora fuckelii (Sacc.) A. Hashim., K. Hiray. \& Kaz. Tanaka, Studies in Mycology 90: 188. 2018. 
Index Fungorum Number: IF551535; Facesoffungi number: FoF00829

Basionym: Lophiostoma fuckelii Sacc., Michelia 1(no. 3): 336 (1878)

= Lophiostoma pulveraceum Sacc., Michelia 1: 336, 1878

= Didymosphaeria lophospora Sacc. \& Speg., Michelia 1: 376, 1878

= Lophiosphaera mendax Rehm, Ann. Myc. 5: 544,1907

= Vaginatispora fuckelii (Sacc.) Thambugala, Fungal diversity 74: 242, 2015

Saprobic on submerged decaying wood in freshwater habitats. Sexual morph: Ascomata 230 $270 \mu \mathrm{m}$ high, $180-220 \mu \mathrm{m}$ diam $(\bar{x}=238 \times 203 \mu \mathrm{m}, \mathrm{n}=5)$, semi-immersed, coriaceous, black, subglobose, ostiolate. Ostiole rounded or slit-like, variable in shape, central, periphysate, with a porelike opening. Peridum 25-40 $\mu \mathrm{m}$ wide, comprise two strata, outer stratum stratum comprising brown to dark brown flattened cells, inner stratum comprising several layers of hyaline cells of textura angularis. Hamathecium comprising 1.5-2.0 $\mu \mathrm{m}$ wide, septate, cellular Pseudoparaphyses, filiform, septate with small guttules, hyaline. Asci $47-54 \times 6.5-8.5 \mu \mathrm{m}(\bar{x}=50 \times 7.5 \mu \mathrm{m}, \mathrm{n}=20), 8$ spored, bitunicate, cylindrical, round at the apex, short pedicellate, with an indistinct ocular chamber. Ascospores 12-13 $\times 3-4 \mu \mathrm{m}(\bar{x}=12 \times 3.5 \mu \mathrm{m}, \mathrm{n}=30)$, biseriate, overlapping, fusiform with acute ends, hyaline, straight or slightly curved, 1-septate, strongly constricted at the septum, mostly 4guttules, smooth-walled, with hyaline appendages at both ends. Asexual morph: Undetermined.

Material examined - CHINA, Yunnan Province, Binchuan county, saprobic on decaying wood submerged in a stream, May 2015, H.W. Shen, S-784 (DLU 784), living culture, MFLUCC 17-1334.

Notes - The new collection is identified as Neovaginatispora fuckelii based on morphology and phylogeny. Morphologically, our new collection fits well with the description of Neovaginatispora fuckelii (Thambugala et al. 2015, Tennakoon et al. 2018). In our phylogenetic analysis, our newly collected isolate clustered with $N$. fuckelii with high bootstrap support (100 ML/1.00 PP). We therefore, identified the newly isolate as $N$. fuckelii.

Neovaginatispora fuckelii has a wide distribution and it has been reported from terrestrial habitats in China, Japan, Germany, Sweden, Switzerland and UK (Wang \& Lin 2004, Thambugala et al. 2015, Tennakoon et al. 2018). In this study, our new collection was collected from a freshwater habitat. Thus, we report our collection as a new record from freshwater habitat.

Vaginatispora armatispora (K.D. Hyde, Vrijmoed, Chinnaraj \& E.B.G. Jones) Wanas., E.B.G. Jones \& K.D. Hyde Stud. Fung. 1(1): 62 (2016)

Fig. 7

Index Fungorum number: IF 819870; Facesoffungi number: FoF 05060

Saprobic on submerged decaying wood in freshwater and marine habitats. Sexual morph: Ascomata 240-340 $\mu \mathrm{m}$ diam. 270-335 $\mu \mathrm{m}$ high, $(\bar{x}=300 \times 288 \mu \mathrm{m}, \mathrm{n}=5)$, scattered, immersed or semi-immersed, globose to subglobose, black, base flatted, coriaceous to carbonaceous. Ostiole black, elongated, crest-like, central, with hyaline periphyses. Peridium 25-40 $\mu \mathrm{m}$ wide, composed of several pale brown to brown cells of textura angularis, cells towards the inside hyaline to pale brown, at the outside, darker, somewhat flattened, fusing and with the host tissues. Hamathecium comprising 1.5-2 $\mu \mathrm{m}$ wide, septate, hyaline pseudoparaphyses composing situated between and above the asci, embedded in a gelatinous matrix. Asci $82-115 \times 13.5-15.7 \mu \mathrm{m}(\bar{x}=108 \times 14.3 \mu \mathrm{m}, \mathrm{n}=20)$, 8 spored, bitunicate, fissitunicate, cylindrical-clavate, short pedicellate, apically rounded with an ocular chamber, hyaline. Ascospores $22-30 \times 5.5-8 \mu \mathrm{m}(\bar{x}=25.8 \times 6.7 \mu \mathrm{m}, \mathrm{n}=30)$, fusiform, with obtuse ends, hyaline when young, pale brown at mature, smooth-walled, 1-septate, strongly constricted at the septum, distinct large guttules, mostly 6-guttules, rarely 4-guttules, 2-3-guttules in each cells, surrounded by a narrow mucilaginous sheath, with distinct hyaline appendages at both ends. Asexual morph: undetermined. 

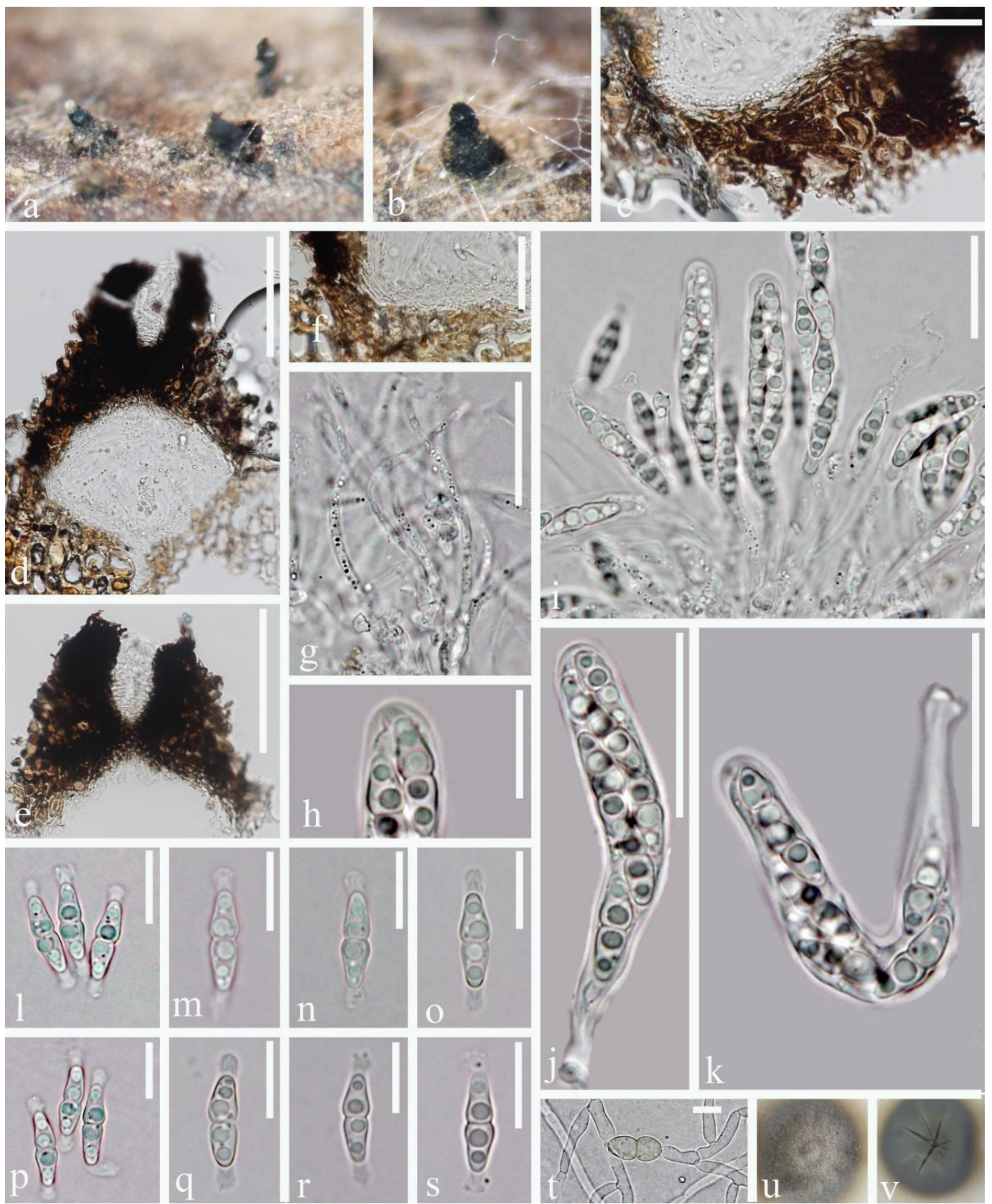

Figure 6 - Neovaginatispora fuckelii (DLU 784) a, b Ascomata on submerged wood. c, f Peridium $\mathrm{d}$ Section of ascoma. e Ostiole. g Pseudoparaphyses. h Ascus apex. i-k Asci. 1-s Ascospores. $t$ Germinating ascospore. $\mathrm{u}, \mathrm{v}$ Culture on PDA. Scale bars: $\mathrm{c}, \mathrm{i}=50 \mu \mathrm{m}, \mathrm{d}, \mathrm{e}=100 \mu \mathrm{m}, \mathrm{f}=30 \mu \mathrm{m}, \mathrm{g}$, $\mathrm{i}-\mathrm{k}=20 \mu \mathrm{m}, \mathrm{h}=5 \mu \mathrm{m}, \mathrm{l}-\mathrm{t}=10 \mu \mathrm{m}$.

Material examined - THAILAND, Sai khu waterfall, Prachuap khiri Khan, on submerged decaying wood, August 2017, V. Kumar, B29, (DLU B29), living culture, MFLUCC 18-0213.

Notes - Vaginatispora armatispora was previously introduced as Massarina armatispora by 
Hyde et al. (1992), that was collected from Mangroves in India and China. The placement of this species was updated by Wanasinghe et al. (2016) and, transferred to Vaginatispora based on both phylogeny and morphology.

In this study, our new isolate (MFLUCC 18-0213) clustered with strains of Vaginatispora armatispora with strong bootstrap (100 ML/1.00 PP). Morphology of our new isolate overlaps with Vaginatispora armatispora (HKTLCC1562, MFLUCC 18-0247) (Hyde et al. 1992, 2019). Therefore, we identified this new isolate as Vaginatispora armatispora.
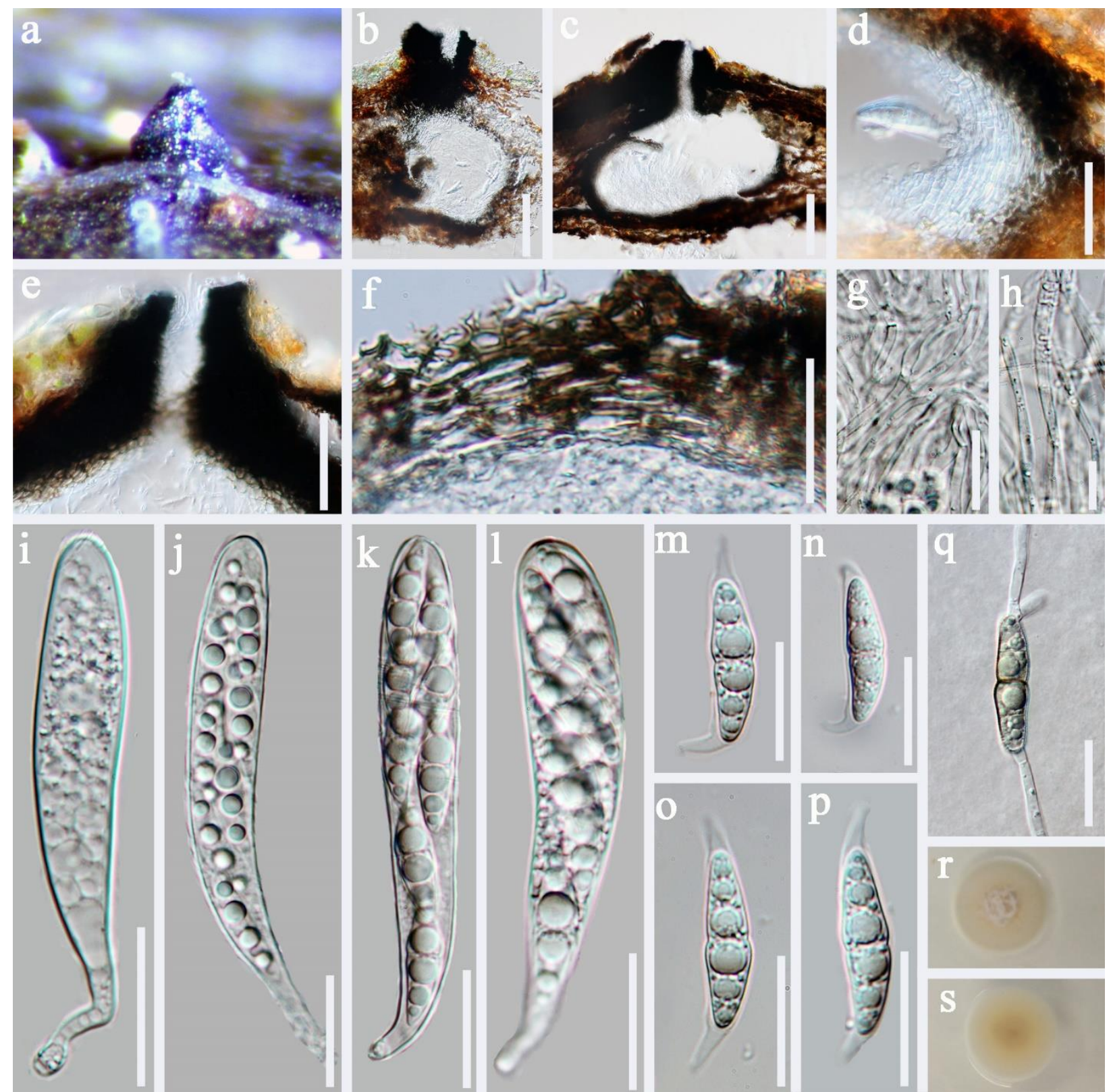

Figure 7 - Vaginatispora armatispora (DLU B29) a Ascomata on submerged wood. b, c Section of ascoma. d, f Peridium. e Ostiole. g, h Pseudoparaphyses. i-l Aci. i-k Asci. m-p Ascospore. q Germinating ascospore. r, s Culture on PDA. Scale bars: $b, c=100 \mu \mathrm{m}, d=30 \mu \mathrm{m}, \mathrm{e}=50 \mu \mathrm{m}, \mathrm{f}, \mathrm{i}-\mathrm{q}$ $=20 \mu \mathrm{m}, \mathrm{g}, \mathrm{h}=10 \mu \mathrm{m}$.

\section{Discussion}

Lophiostomataceae, with over 150 species, is a highly speciose family in Pleosporales, 
(Wijayawardene et al. 2018). They are cosmopolitan and distributed in many countries (China, France, Hungary, Italy, India, Japan, Russia and Thailand) (Thambugala et al. 2015, Wijayawardene et al. 2017, Hashimoto et al. 2018, Wanasinghe et al. 2018). However, only two Lophiostomataceae species viz. Lophiostoma bipolare and L. proprietunicatum have been reported in Yunnan, China (Luo et al. 2004). In this study, we carried out research on lignicolous freshwater fungi in Tibet and Yunnan provinces, China and Southern Thailand. Six Lophiostomataceae species have been collected and among them, five species are reported from China showing that members of this family are also widely distributed in China.

Species of Lophiostomataceae are highly diverse in their ascomata, peridium, pseudoparaphyses and ascospores characters. It is difficult to distinguish the species based on morphological characters alone and many species lack sequence data (Thambugala et al. 2015). Furthermore, some genera in Lophiostomataceae such as Lophiostoma are paraphyletic (Hashimoto et al. 2018). Therefore, further collections and phylogenetic studies are recommended for better understand the species boundaries within these genera.

Flabellascoma species are so far reported from terrestrial habitats (Hashimoto et al. 2018), while the two new species, $F$. aquaticum and $F$. fusiforme were collected from freshwater. This expands the habitat range of Flabellascoma species to freshwater. Species of Flabellascoma have similar shape and size of asci and ascospores and it is therefore difficult to distinguish them. but ascomatal features appear to be informative to delineate them. Flabellascoma aquaticum ascomata has a long black neck, whereas, $F$. fusiforme has ascomata with a short, elongated, crest-like ostiolar neck (Hashimoto et al. 2018). Sigarispora is a well-resolved genus in Lophiostomataceae. Species in this genus are known from terrestrial habitats in Russia, Italy and Japan (Li et al. 2016, Thambugala et al. 2015, Wanasinghe et al. 2018) and in this study, the new species Sigarispora clavata is reported from freshwater habitats in China.

\section{Acknowledgements}

We would like to thank the National Natural Science Foundation of China (Project ID: 31660008 and 31860006), Fungal diversity conservation and utilization innovation team of Dali University (ZKLX2019213) and the Thailand Research Fund grant "impact of climate change on fungal diversity and biogeography in the Greater Mekong Sub-region (RDG6130001)" for financial and laboratory support. Dan-Feng Bao thanks Shaun Pennycook from Landcare Research, Auckland, New Zealand, for advising on the taxon names, Wen-Li Li, Yan-Mei Zhang and Hong-Wei Shen are acknowledged for their help on DNA extraction and PCR amplification.

\section{References}

Ariyawansa HA, Hyde KD, Jayasiri SC, Buyck B et al. 2015 - Fungal diversity notes 111-252taxonomic and phylogenetic contributions to fungal taxa. Fungal Diversity 75, 27-274.

Bucher VVC, Hyde KD, Pointing SB, Reddy CA. 2004 - Production of wood decay enzymes, mass loss and lignin solubilization in wood by diverse freshwater fungi. Fungal Diversity 15, 1-14.

Chomnunti P, Hongsanan S, Aguirre-Hudson B, Tian Q et al. 2014 - The sooty moulds. Fungal Diversity 66, 1-36.

Glez-Peña D, Gómez-Blanco D, Reboiro-Jato M, Fdez-Riverola F et al. 2010 - ALTER: programoriented conversion of DNA and protein alignments. Nucleic Acids Research 38, 14-18.

Hall TA. 1999 - BioEdit: a user-friendly biological sequence alignment editor and analysis program for Windows 95/98/NT. Nucleic Acids Symposium Series 41, 95-98. 
Hashimoto A, Hirayama K, Takahashi H, Matsumura M et al. 2018 - Resolving the Lophiostoma bipolare complex: Generic delimitations within Lophiostomataceae. Studies in Mycology 90, 161-189.

Ho WH, Yanna, Hyde KD, Hodgkiss IJ. 2002 - Seasonality and sequential occurrence of fungi on wood submerged in Tai Po Kau Forest Stream, Hong Kong. Aquatic Microbial Ecology 21, 289-298.

Hyde KD. 1992 - Tropical Australian freshwater fungi. I. Some ascomycetes. Austral Syst Bot 5, 109-116.

Hyde KD. 1995 - Tropical Australia freshwater fungi VII. New genera and species of ascomycetes. Nova Hedwigia 61, 119-140.

Hyde KD, Goh TK 1998 - Fungi on submerged wood in Lake Barrine, north Queensland, Australia. Mycological Research 102, 739-749.

Hyde KD, Fryar S, Tian Q, Bahkali AH et al. 2016 - Lignicolous freshwater fungi along a northsouth latitudinal gradient in the Asian/Australian region; can we predict the impact of global warming on biodiversity and function? Fungal Ecology 19, 190-200.

Hyde KD, Jones EBG, Liu JK, Ariyawansa H. 2013 - Families of Dothideomycetes. Fungal Diversity $63,1-313$.

Hyde KD, Norphanphoun C, Abreu VP, Bazzicalupo A et al. 2017 - Fungal diversity notes 603-708: taxonomic and phylogenetic notes on genera and species. Fungal Diversity 87, 1-235.

Hyde KD, Tennakoon DS, Jeewon R, Bhat DJ et al. 2019 - Fungal diversity notes 1036-1150: taxonomic and phylogenetic contributions on genera and species of fungal taxa. Fungal Diversity 96, 1-242.

Jayasiri SC, Hyde KD, Ariyawansa HA, Bhat DJ et al. 2015 - The faces of fungi database: fungal names linked with morphology, phylogeny and human impacts. Fungal Diversity 74, 3-18.

Katoh K, Standley DM. 2013 - MAFFT multiple sequence alignment software version 7: improvements in performance and usability. Molecular biology and evolution 30, 772-780.

Kodsueb R, Vijaykrishna D, Aptroot A, Lumyong S et al. 2006 - The family Pleosporaceae: intergeneric relationships and phylogenetic perspectives based on sequence analyses of partial 28S rDNA. Mycologia 98, 571-583.

Li GJ, Hyde KD, Zhao RL, Hongsanan S et al. 2016 - Fungal diversity notes 253-366: taxonomic and phylogenetic contributions to fungal taxa. Fungal Diversity 78, 1-237.

Liu JK, Hyde KD, Jones EG, Ariyawansa HA et al. 2015 - Fungal diversity notes 1-110: taxonomic and phylogenetic contributions to fungal species. Fungal Diversity 72, 1-197.

Liu YJ, Whelen S, Hall BD. 1999 - Phylogenetic relationships among ascomycetes: evidence from an RNA polymerase II subunit. Molecular Biology and Evolution 16, 1799-1808.

Luo J, Yin J, Cai L, Zhang K et al. 2004 - Freshwater fungi in Lake Dianchi, a heavily polluted lake in Yunnan, China. Fungal Diversity 16, 93-112.

Luo ZL, Hyde, KD, Liu JK, Bhat DJ et al. 2018 - Lignicolous freshwater fungi from China II: Novel Distoseptispora (Distoseptisporaceae) species from northwestern Yunnan Province and a suggested unified method for studying lignicolous freshwater fungi. Mycosphere 9, 444-461.

Miller MA, Pfeiffer W, Schwartz T. 2010 - Creating the CIPRES Science Gateway for inference of large phylogenetic trees. In: Proceedings of the 2010 Gateway Computing Environments Workshop (GCE), New Orleans, Louisiana, November 2010, 1-8.

Nitschke TRJ. 1869 - Grundlage eines systems der Pyrenomyceten. Verhandlungen des Naturhistorischen Vereins der Preussischen Rheinlande. Westfalens und des Regierungsbezirks Osnabrück 26, 70-77. 
Nylander JAA. 2004 - MrModeltest v2 Program distributed by the author. Evolutionary Biology Centre, Uppsala University, Uppsala.

Palmer ME, Covich AP, Finlay BJ, Gilbert J et al. 1997 - Biodiversity and ecosystem processes in freshwater sediments. Ambio 26, 571-577.

Rannala B, Yang Z. 1996 - Probability distribution of molecular evolutionary trees: a new method of phylogenetic inference. Journal of Molecular Evolution 43, 304-311.

Ronquist F, Teslenko M, van der Mark P, Ayres DL et al. 2012 - MrBayes 3.2: efficient Bayesian phylogenetic inference andmodel choice across a large model space. Systematic Biology 61, 539-542.

Saccardo PA. 1883 - Sylloge Fungorum Omnium hucusque cognitorum: Volume 2. Padova, Italy.

Stamatakis A, Hoover P, Rougemont J. 2008 - A rapid bootstrap algorithm for the RAxML webservers. Systematic Biology 75, 758-771.

Stamatakis A. 2006 - RAxML-VI-HPC: maximum likelihood-based phylogenetic analyses with thousands of taxa and mixed models. Bioinformatics 22, 2688-2690.

Tennakoon DS, Kuo CH, Jeewon R, Thambugala KM et al. 2018 - Saprobic Lophiostomataceae (Dothideomycetes): Pseudolophiostoma mangiferae sp. nov. and Neovaginatispora fuckelii, a new record from Mangifera indica. Phytotaxa 364, 157-17.

Thambugala KM, Hyde KD, Tanaka K, Tian Q et al. 2015 - Towards a natural classification and backbone tree for Lophiostomataceae, Floricolaceae, and Amorosiaceae fam. nov. Fungal Diversity 74, 199-266.

Tibpromma S, Hyde KD, Jeewon R, Maharachchikumbura SSN et al. 2017 - Fungal diversity notes 491-602: taxonomic and phylogenetic contributions to fungal taxa. Fungal Diversity 83, 1261.

Vilgalys R, Hester M. 1990 - Rapid genetic identification and mapping of enzymatically amplified ribosomal DNA from several Cryptococcus species. Journal of Bacteriology 172, 4238-4246.

Wanasinghe DN, Jones EBG, Dissanayake AJ, Hyde KD 2016 - Saprobic Dothideomycetes in Thailand: Vaginatispora appendiculata sp. nov. (Lophiostomataceae) introduced based on morphological and molecular data. Studies in Fungi 1, 56-68.

Wanasinghe DN, Phukhamsakda C, Hyde KD, Jeewon R et al. 2018 - Fungal diversity notes 709839: taxonomic and phylogenetic contributions to fungal taxa with an emphasis on fungi on Rosaceae. Fungal Diversity 89, 1-236.

Wang HK, Aproot A, Crous PW, Hyde KD et al. 2007 - The polyphyletic nature of Pleosporales: An example from Massariosphaeria based on ribosomal DNA and RBP2 gene phylogenies. Fungal Biology 111, 1268-1276.

White TJ, Bruns T, Lee S, Taylor J. 1990 - Amplification and direct sequencing of fungal ribosomal RNA genes for phylogenetics. In: Innis GM, Shinsky D, White T (eds) PCR protocols: a guide to methods and applications. Academic, New York, 315-322.

Wijayawardene NN, Hyde KD, Lumbsch TH, Liu JK et al. 2018 - Outline of Ascomycota: 2017. Fungal Diversity 88, 167-263.

Wijayawardene NN, Hyde KD, Rajeshkumar KC, Hawksworth et al. 2017 - Notes for genera: Ascomycota. Fungal Diversity 86, 1-594.

Wong KMK, Goh TK, Hodgkiss IJ, Hyde KD et al. 1998 - Role of fungi in freshwater ecosystems. Biodiversity, Conservation 7, 1187-1206. 